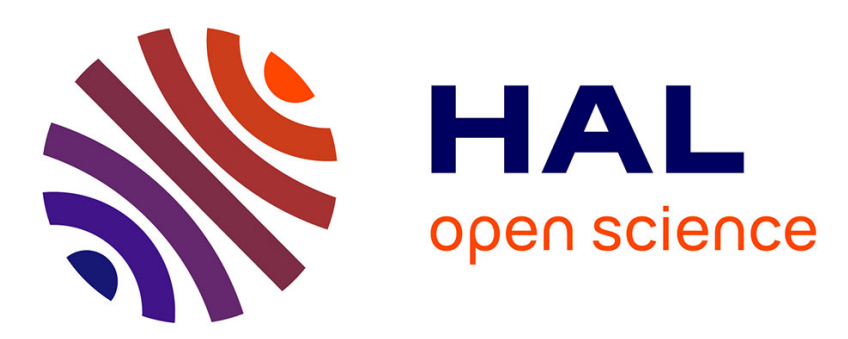

\title{
Technique originale de piézo-réflexion-rétrotransmission pour la détermination et l'identification simultanées des gaps direct et indirect d'un semiconducteur
}

\author{
P. Merle, Daniel Auvergne, H. Mathieu
}

\section{- To cite this version:}

P. Merle, Daniel Auvergne, H. Mathieu. Technique originale de piézo-réflexion-rétrotransmission pour la détermination et l'identification simultanées des gaps direct et indirect d'un semiconducteur. Revue de Physique Appliquée, 1977, 12 (1), pp.71-75. 10.1051/rphysap:0197700120107100 jpa-00244122

HAL Id: jpa-00244122

https://hal.science/jpa-00244122

Submitted on 1 Jan 1977

HAL is a multi-disciplinary open access archive for the deposit and dissemination of scientific research documents, whether they are published or not. The documents may come from teaching and research institutions in France or abroad, or from public or private research centers.
L'archive ouverte pluridisciplinaire HAL, est destinée au dépôt et à la diffusion de documents scientifiques de niveau recherche, publiés ou non, émanant des établissements d'enseignement et de recherche français ou étrangers, des laboratoires publics ou privés. 


\title{
TECHNIQUE ORIGINALE DE PIÉZO-RÉFLEXION-RÉTROTRANSMISSION POUR LA DÉTERMINATION ET L'IDENTIFICATION SIMULTANÉES DES GAPS DIRECT ET INDIRECT D'UN SEMICONDUCTEUR
}

\author{
P. MERLE, D. AUVERGNE et H. MATHIEU \\ Centre d'Etudes d'Electronique des Solides (*) \\ Université des Sciences et Techniques du Languedoc, 34060 Montpellier Cedex, France
}

(Reçu le 15 juin 1976, accepté le 28 juillet 1976)

\begin{abstract}
Résumé. - Compte tenu des propriétés spécifiques de la piézomodulation, nous montrons comment une technique expérimentale originale, la piézo-réflexion-rétrotransmission, permet sur un même spectre d'observer, d'identifier et de positionner en énergie le gap direct et le gap indirect d'un matériau et de préciser la nature de ce gap indirect $(L$ ou $X)$.
\end{abstract}

\begin{abstract}
Using the properties of the piezomodulation and an original experimental technique, it is possible to observe in the same spectrum the structures characteristic of the direct gap and those of the indirect one. It is shown that it is possible to identify the nature of the indirect gaps, owing to the different values of the deformation potential.
\end{abstract}

1. Introduction. - En raison de leurs applications industrielles pour la réalisation de dispositifs électroniques tels que diodes laser, diodes Gunn, diodes électroluminescentes, ou convertisseurs d'énergie solaire les composés III-V et leurs alliages ont connu un développement considérable au cours des dernières années. Le rendement optimal de ces dispositifs est lié à la nature des minima de conduction mis en jeu dans les processus physiques considérés. La connaissance de l'évolution, en fonction de la composition, de la séparation en énergie des minima de conduction $\Gamma, L$ et $X$ apparaît donc d'une importance fondamentale. En particulier dans les alliages réalisés à partir de composés extrêmes ayant des seuils respectivement direct et indirect, il est possible de prévoir un seuil fondamental faisant intervenir, successivement les différents minima de conduction, suivant la composition de l'alliage. Dans ce cas la détermination précise des valeurs de la composition correspondant au croisement des différents minima est très importante aussi bien du point de vue pratique que du point de vue fondamental.

Pour atteindre cet objectif, il devient nécessaire

(*) Associé au C. N. R. S. d'utiliser une technique de mesure permettant de distinguer sans ambiguïté un gap direct d'un gap indirect et dans le deuxième cas de pouvoir préciser la nature du minimum de conduction mise en jeu ( $X$ ou $L)$.

Le problème de la détermination d'un gap indirect a été extensivement étudié, et les méthodes de réflectivité différentielle s'avèrent particulièrement bien adaptées [1]. En ce qui concerne un gap indirect, la réflectivité différentielle ne donne aucun résultat car la force d'oscillateur de ce type de transition est trop faible pour induire les structures observables sur la partie réelle $\varepsilon_{1}$ de la constante diélectrique. La partie imaginaire $\varepsilon_{2}$, présente par contre au voisinage de chaque seuil une variation en énergie de la forme $\left(E-E_{\mathrm{s}}\right)^{1 / 2}$ lorsque la transition est excitonique. Il en résulte que sa dérivée présente une discontinuité à chaque seuil ce qui rend les techniques de transmission différentielle particulièrement bien adaptées à l'étude de ces transitions [2, 3, 4].

Un problème demeure cependant dans l'étude des alliages, c'est l'identification du minimum de la bande de conduction $\left(X_{1 \mathrm{c}}\right.$ ou $\left.L_{1 \mathrm{c}}\right)$ concerné dans les transitions indirectes. Or les minima $X$ et $L$ de la bande de conduction ayant des potentiels de déformation hydrostatique de signes opposés, la technique de trans- 
mission différentielle la mieux adaptée à l'identification de ces minima est la piézotransmission. C'est la technique que nous avons choisie.

Nous présentons ici une technique expérimentale originale permettant sur un même spectre, d'observer, d'identifier et de positionner en énergie, le gap direct et le gap indirect du matériau et de préciser la nature de ce gap indirect $\left(\Gamma_{15 \mathrm{v}} \rightarrow X_{1 \mathrm{c}}\right.$ ou $\left.\Gamma_{15 \mathrm{v}} \rightarrow L_{1 \mathrm{c}}\right)$.

2. Considérations théoriques sur la modulation d'un gap fondamental. - Dans un matériau à structure blende les transitions directes et indirectes sont dans la notation du simple groupe :

$$
\Gamma_{15 \mathrm{v}} \rightarrow \Gamma_{1 \mathrm{c}} \quad \Gamma_{15 \mathrm{v}} \rightarrow X_{1 \mathrm{v}} \quad \Gamma_{15 \mathrm{v}} \rightarrow L_{1 \mathrm{c}} .
$$

Les premières sont observables dans les composés III-V directs, InP, GaSb, GaAs, ..., les secondes dans les composés III-V indirects, tels que AlP, AlAs, AlSb, $\mathrm{GaP}$, et les troisièmes dans certains alliages pseudobinaires, tels que $\mathrm{Ga}_{1-x} \mathrm{Al}_{x} \mathrm{Sb}$ ou $\mathrm{Ga}_{x} \mathrm{In}_{1-x} \mathrm{P}$ qui présentent la particularité d'avoir les trois minimas $\Gamma, L$ et $X$ très voisins, pour des valeurs de $x$ voisines respectivement de 0,4 et de 0,7.

Les transitions indirectes, sont caractérisées par l'existence d'une transition directe sur un état intermédiaire, et par la diffusion électron-phonon de cet état intermédiaire vers l'état final (ou de l'état initial vers ce même état intermédiaire). Les états intermédiaires d'une part, et les phonons d'autre part, mis en jeu dans ces diverses transitions, sont déterminés simplement à l'aide de la théorie des groupes [2]. L'état intermédiaire le plus probable est l'état $\Gamma_{1 \mathrm{c}}$, le plus proche de l'état final $\left(X_{1 \mathrm{c}}\right.$ ou $\left.L_{1 \mathrm{c}}\right)$, les symétries des phonons en bord de zone sont :

$$
\begin{gathered}
X=X_{5}(\mathrm{TO})+X_{5}(\mathrm{TA})+X_{1}(\mathrm{LA} \mathrm{ou} \mathrm{LO})+ \\
+X_{3}(\mathrm{LO} \text { ou LA }) \\
L=L_{2}(\mathrm{TO})+L_{3}(\mathrm{TA})+L_{1}(\mathrm{LA})+L_{1}(\mathrm{LO}) .
\end{gathered}
$$

$X_{3}$ est la symétrie de la vibration longitudinale de l'atome choisi comme origine $x_{1}=(000)$, et $X_{1}$ la symétrie de la vibration longitudinale de l'atome situé en $x_{2}=a\left(\frac{1}{4}, \frac{1}{4}, \frac{1}{4}\right)$. En prenant, comme pour le calcul de la structure des bandes d'énergie électroniques, l'origine sur l'atome du groupe $\mathrm{V}, X_{1}$ est la symétrie de la vibration longitudinale de l'atome III, et $X_{3}$ la symétrie de la vibration longitudinale de l'atome $\mathrm{V}$. Ainsi $X_{1}$ est la symétrie du phonon LA (et $X_{3}$ celle du phonon LO), dans les matériaux dans lesquels l'atome du groupe III est plus lourd que l'atome du groupe V, et inversement. Il en résulte que dans le GaP les symétries des phonons longitudinaux sont $X_{1}(\mathrm{LA})$ et $X_{3}(\mathrm{LO})$, et que, par contre, dans AlP, AlAs et AlSb ces symétries sont inversées, $X_{1}(\mathrm{LO})$ et $X_{3}(\mathrm{LA})$. On peut ainsi montrer [6] que la transition $\Gamma_{15 \mathrm{v}} \rightarrow X_{1 \mathrm{c}}$, mettant en jeu l'état intermédiaire $\Gamma_{1 \mathrm{c}}$, de loin la plus probable, n'est possible que par l'intermédiaire d'un phonon $\mathrm{LA}_{x}(\mathrm{GaP})$ ou $\mathrm{LO}_{x}$ (AlSb, AlAs, AlP) et que la transition $\Gamma_{15 \mathrm{v}} \rightarrow L_{1 \mathrm{c}}$, mettant en jeu l'état intermé- diaire $\Gamma_{1 \mathrm{c}}$, est possible par l'intermédiaire des phonons $\mathrm{LA}_{l}$ et $\mathrm{LO}_{l}$.

Pour chaque processus de diffusion et pour un phonon déterminé, le coefficient d'absorption s'écrit sous la forme générale [7] :

$$
\alpha \sim \frac{P^{2} H^{2}}{\left(\delta E \pm \hbar \omega_{\mathrm{p}}\right)^{2}} f(E) .
$$

$P$ est l'élément de matrice de la transition verticale dû au couplage électron photon,

$H$ est l'élément de matrice de la diffusion électron phonon,

$\delta E$ est la différence d'énergie entre l'état intermédiaire et l'état final, ou entre l'état initial et l'état intermédiaire, $\hbar \omega_{\mathrm{p}}$ l'énergie du phonon,

le signe \pm ayant trait soit à l'absorption soit à l'émission du phonon,

$f(E)$ représente la variation en fonction de l'énergie du coefficient d'absorption, qui s'écrit suivant le type de transition :

$$
\begin{aligned}
& f(E)=\left(E-E_{\mathrm{g}} \pm \hbar \omega_{\mathrm{p}}\right)^{2} \\
& \text { Transition permise sans exciton } \\
& f(E)=\left(E-E_{\mathrm{g}} \pm \hbar \omega_{\mathrm{p}}+E_{\mathrm{ex}}\right)^{1 / 2} \\
& \text { Transition permise avec exciton } \\
& f(E)=\left(E-E_{\mathrm{g}} \pm \hbar \omega_{\mathrm{p}}\right)^{3} \\
& \text { Transition interdite sans exciton } \\
& f(E)=\left(E-E_{\mathrm{g}} \pm \hbar \omega_{\mathrm{p}}\right)^{3 / 2}
\end{aligned}
$$

Transition interdite avec exciton.

La présence, au dénominateur du terme $\delta E^{2}$ montre que les transitions mettant en jeu l'état intermédiaire $\Gamma_{1 \mathrm{c}}$ auront un poids bien supérieur aux autres, en raison des valeurs relatives des énergies $\Gamma_{1 \mathrm{c}}-X_{1 \mathrm{c}}$ ou $\Gamma_{1 \mathrm{c}}-L_{1 \mathrm{c}}$ d'une part, et $\Gamma_{15 \mathrm{c}}-X_{1 \mathrm{c}}$ et $\Gamma_{15 \mathrm{v}}-X_{5 \mathrm{v}}$, ou $\Gamma_{15 \mathrm{c}}-L_{1 \mathrm{c}}$ et $\Gamma_{15 \mathrm{v}}-L_{3 \mathrm{v}}$ d'autre part.

Lorsqu'on applique une contrainte uniaxiale ou coplanaire au matériau le sommet $\Gamma_{15 \mathrm{v}}$ de la bande de valence se dédouble et se déplace, ce qui entraîne une double modulation du niveau inital. De la même manière, les minima $\Gamma_{1 \mathrm{c}}, X_{1 \mathrm{c}}$ ou $L_{1 \mathrm{c}}$ se déplacent mais sans se dédoubler, en effet ce sont des minima à une dimension, et la contrainte ne lève pas la dégénérescence de spin, par contre les 3 ellipsoïdes $X_{1 \mathrm{c}}$, ou les 4 ellipsoïdes $L_{1 \mathrm{c}}$, qui sont équivalents dans le cristal non perturbé, sont différemment modulés par la contrainte suivant la symétrie de cette dernière. Il en résulte d'une manière générale, une double modulation du gap direct $\Gamma_{15 \mathrm{v}} \rightarrow \Gamma_{1 \mathrm{c}}$ et une quadruple modulation des gaps indirects, $\Gamma_{15 \mathrm{v}} \rightarrow X_{1 \mathrm{c}}$ et $\Gamma_{15 \mathrm{v}} \rightarrow L_{1 \mathrm{c}}$. De plus les différentes transitions élémentaires sont non seulement modulées différemment, mais aussi régies par des éléments de matrice différents. Enfin, pour un gap déterminé, les différentes transitions élémentaires mettent en jeu des états initiaux, issus du dédoublement par la contrainte de la bande de valence $\Gamma_{15 \mathrm{v}}$, de densités différentes.

Ainsi pour calculer le paramètre de modulation d'un gap déterminé, direct ou indirect, nous devons effectuer 
une somme pondérée des différentes transitions élémentaires. Les quatre paramètres de pondération de chaque transition étant, son amplitude de modulation, son élément de matrice, la densité d'état du niveau initial, le nombre d'ellipsoïdes de conduction mis en jeu.

Nous avons précédemment calculé ces paramètres de modulation pour des contraintes uniaxiales et coplanaires à symétrie $(001)$ et (111), pour tous les types de phonons mis en jeu, pour tous les états intermédiaires concernés et pour les deux types de polarisation $\pi$ et $\sigma$. Ces calculs sont développés dans la référence [6].

Dans le cas d'une piézomodulation par contrainte coplanaire (111) ou (001) que nous utiliserons dans nos expériences les paramètres de modulation des différents seuils sont [6] :

$$
\begin{gathered}
\Delta E_{\mathrm{g}}^{\Gamma}=\frac{1}{4} \frac{1}{1+\gamma}\left(2 \alpha_{\mathrm{d}}+\theta\right)+ \\
\quad+\frac{3}{4} \frac{\gamma}{1+\gamma}\left(2 \alpha_{\mathrm{d}}-\theta\right) \\
\Delta E_{\mathrm{g}}^{x}\left(\mathrm{LA}_{X}\right)=\frac{1}{4} \frac{1}{1+\gamma}\left(2 \alpha_{x}+\theta\right)+ \\
+\frac{3}{4} \frac{\gamma}{1+\gamma}\left(2 \alpha_{x}-\theta\right)
\end{gathered}
$$

$$
\begin{aligned}
\Delta E_{\mathrm{g}}^{1}\left(\mathrm{LA}_{L}, \mathrm{LO}_{L}\right)=\frac{1}{4} \frac{1}{1+\gamma} & \left(2 \alpha_{l}+\theta\right)+ \\
& +\frac{3}{4} \frac{\gamma}{1+\gamma}\left(2 \alpha_{l}-\theta\right)
\end{aligned}
$$

où $\quad \alpha_{\mathrm{d}}=a_{\mathrm{d}}\left(S_{11}+2 S_{12}\right) ; \quad \alpha_{x}=a_{i x}\left(S_{11}+2 S_{12}\right)$; $\alpha_{l}=a_{i l}\left(S_{11}+2 S_{12}\right), \quad \theta=\beta=b\left(S_{11}-S_{12}\right)$ pour une contrainte (001),

$$
\theta=\delta=\frac{d}{2 \sqrt{3}} S_{44}
$$

pour une contrainte (111). Les $S_{i j}$ sont les coefficients d'élasticité. $a_{d}, a_{i x}, a_{i l}, b$ et $d$ sont les potentiels de déformations. $\gamma=\left(m_{1} / m_{2}\right)^{3 / 2}$ tient compte des densités d'état différentes des bandes de valence dégénérées en $\Gamma_{15 v^{\circ}}$.

Dans le cas de GaP et AlSb qui sont les seuls composés III-V pour lesquels les potentiels de déformation des gaps indirects ont été mesurés, on obtient pour ces différents paramètres les valeurs portées dans le tableau I. Les valeurs numériques portées dans ce

\section{TABleaU I}

Paramètres de piézomodulation (en $10^{-6} \mathrm{eV} / \mathrm{bar}$ ) des différentes transitions fondamentales pour deux types de contraintes coplanaires (001) et (111).

\begin{tabular}{lcccccc} 
& \multicolumn{2}{c}{$\Delta E_{\mathrm{g}}^{\Gamma}$} & \multicolumn{2}{c}{$\Delta E_{\mathrm{g}}^{x}(\mathrm{LA})$} & \multicolumn{2}{c}{$\Delta E_{\mathrm{g}}^{l}(\mathrm{LA}, \mathrm{LO})$} \\
& $(001)$ & $-(111)$ & $(001)$ & $(111)$ & $(001)$ & $(111)$ \\
$\mathrm{GaP}$ & 6,07 & 5,9 & $-0,66$ & $-0,8$ & 2,52 & 2,4 \\
$\mathrm{AlSb}$ & 6,49 & 6,5 & 0,19 & 0,21 & 3,05 & 3
\end{tabular}

tableau ne sont qu'indicatives compte tenu essentiellement du fait que la valeur numérique du potentiel de déformation $a_{i l}$ est une valeur calculée. Par contre, les signes de ces paramètres sont significatifs. Dans le but d'étudier ces signes, il est possible de simplifier les expressions $(2 a, b, c)$ compte tenu de l'ordre de grandeur des différents coefficients et en particulier $3 \gamma \gg 1$. On obtient alors :

$$
\begin{aligned}
\Delta E_{\mathrm{g}}^{\Gamma} & \simeq 2 \alpha_{d}-\theta \\
\Delta E_{\mathrm{g}}^{x}\left(\mathrm{LA}_{X}\right) & \simeq 2 \alpha_{x}-\theta \\
\Delta E_{\mathrm{g}}^{l}\left(\mathrm{LA}_{L}, \mathrm{LO}_{L}\right) & \simeq 2 \alpha_{l}-\theta .
\end{aligned}
$$

Considérons successivement chacun des trois paramètres :

$\Delta E_{\mathrm{g}}^{\Gamma}$ : les potentiels de déformation $a_{\mathrm{d}}, b$ et $d$ étant connus expérimentalement sans ambiguïté, on peut admettre comme acquis le signe positif de $\Delta E_{\mathrm{g}}^{\Gamma}$.

$\Delta E_{\mathrm{g}}^{x}\left(\mathrm{LA}_{X}\right)$ : De même les paramètres $a_{i x}, b$ et $d$ étant mesurés expérimentalement on peut admettre comme acquis le signe négatif de $\Delta E_{\mathrm{g}}^{\boldsymbol{x}}\left(\mathrm{LA}_{X}\right)$ dans $\mathrm{GaP}$ et son signe positif dans AlSb.

$\Delta E_{\mathrm{g}}^{l}\left(\mathrm{LO}_{L}, \mathrm{LA}_{L}\right)$ : Il faut noter ici que $\theta$ est négatif car $b$ et $d$ sont négatifs. D'autre part, $\alpha_{l}$ est toujours positif, en effet les calculs théoriques montrent que $a_{i l}$ est positif dans tous les semiconducteurs [8], d'autre part les mesures expérimentales effectuées sur $\mathrm{Ge}$ donnent aussi des résultats positifs. Il en résulte que ce paramètre de modulation est toujours positif.

La conclusion de cette discussion est que tous les gaps $\left(\Gamma_{15 \mathrm{v}} \rightarrow \Gamma_{1 \mathrm{c}}, \Gamma_{15 \mathrm{v}} \rightarrow X_{1 \mathrm{c}}\right.$ et $\left.\Gamma_{15 \mathrm{v}} \rightarrow L_{1 \mathrm{c}}\right)$ sont modulés en phase dans AlSb, alors que dans GaP le gap $\Gamma_{15 \mathrm{v}} \rightarrow X_{1 \mathrm{c}}$ est modulé en opposition de phase avec les deux autres. Ce résultat très important permet d'identifier les transitions indirectes en $X$ des transitions indirectes en $L$.

Avant d'aller plus loin, il est important de noter que les résultats obtenus sur les monocristaux peuvent être étendus aux polycristaux. Ceci est d'une importance déterminante pour l'étude des alliages, car ces derniers sont souvent obtenus sous forme polycristalline.

Dans les polycristaux, on peut définir un nouveau milieu isotrope par compensation, caractérisé par des coefficients d'élasticité moyens donnés dans les matériaux cubiques par [9]:

$$
\begin{aligned}
& \bar{S}_{11}=\frac{3}{5} S_{11}+\frac{2}{5} S_{12}+\frac{1}{5} S_{44} \\
& \bar{S}_{12}=\frac{1}{5} S_{11}+\frac{4}{5} S_{12}-\frac{1}{10} S_{44} \\
& \bar{S}_{44}=\frac{4}{5} S_{11}-\frac{4}{5} S_{12}+\frac{3}{5} S_{44} .
\end{aligned}
$$

Dans le cas d'une contrainte coplanaire arbitraire 
((11) par exemple), les valeurs moyennes des coefficients $\alpha$ et $\delta$ sont données par :

$$
\begin{aligned}
& \bar{\delta}=\bar{a}_{i}\left(\bar{S}_{11}+2 \bar{S}_{12}\right) \\
& \bar{\delta}=\frac{\bar{d}}{2 \sqrt{3}} \bar{S}_{44} .
\end{aligned}
$$

Or $d$, est le potentiel de déformation pour un cisaillement pur de sorte que $\bar{d}=0$, d'autre part $a_{i}$, qui est une composante hydrostatique permet d'écrire $\bar{a}_{i}=a_{i}$ de sorte que le résultat s'écrit :

$$
\bar{\alpha}=\alpha \quad \text { et } \quad \bar{\delta}=0
$$

donc

$$
\frac{\Delta E_{\mathrm{g}}^{x}\left(\mathrm{LA}_{X}\right)}{\Delta E_{\mathrm{g}}^{l}\left(\mathrm{LA}_{L}, \mathrm{LO}_{L}\right)}=\frac{\alpha_{x}}{\alpha_{l}}=\frac{a_{i x}}{a_{i l}}
$$

Ce rapport est négatif puisque dans tous les matériaux $a_{i x}$ est négatif et $a_{i l}$ est positif.

Il en résulte que dans le cas d'alliages polycristallins, il est toujours possible de distinguer les transitions en $X$ des transitions en $L$, alors que, nous venons de le voir, ceci n'est pas possible dans les monocristaux de AlSb par exemple.

3. Technique expérimentale. - La technique de spectroscopie différentielle par piézomodulation, consiste à moduler les constantes optiques d'un échantillon au moyen d'un transducteur piézoélectrique excité par un générateur de puissance basse fréquence. Le signal réfléchi ou transmis prélevé à la sortie du détecteur, est analysé par une détection synchrone, un ratiomètre effectue ensuite le rapport $\Delta R / R$ qui est transmis à un enregistreur.

La mise à profit des résultats résumés dans le tableau I, nécessite la connaissance de la phase du signal mesuré, or il n'est pas possible de connaître en valeur absolue la phase d'un signal issu d'une détection synchrone puisque ce signal est défini modulo $\pi$. Par contre il est possible sur un même enregistrement de comparer la phase de deux signaux. Ainsi dans le cas qui nous intéresse nous prendrons la phase du signal de piézoréflectance sur le gap direct $(\Delta R / R)_{\Gamma}$ comme référence et nous comparerons à ce signal le signal de piézotransmission au niveau du gap indirect $(\Delta T / T)_{\text {ind }}$. Il est alors nécessaire d'enregistrer ces deux signaux sur un même spectre. Nous avons dans ce but mis au point une technique de piézo-réflexion-rétrotransmission qui consiste à mesurer le spectre de piézoréflectivité d'un échantillon mince ayant deux faces polies (Fig. 1).

La réflexion sur la face avant donne une structure caractéristique de la partie réelle de la constante diélectrique, sensible aux niveaux des gaps fondamentaux uniquement à un gap direct soit :

$$
(\Delta R / R)_{\mathrm{av}}=\alpha \Delta \varepsilon_{1} \simeq \frac{\mathrm{d} \varepsilon_{1}}{\mathrm{~d} E} \Delta E_{\mathrm{direct}}
$$

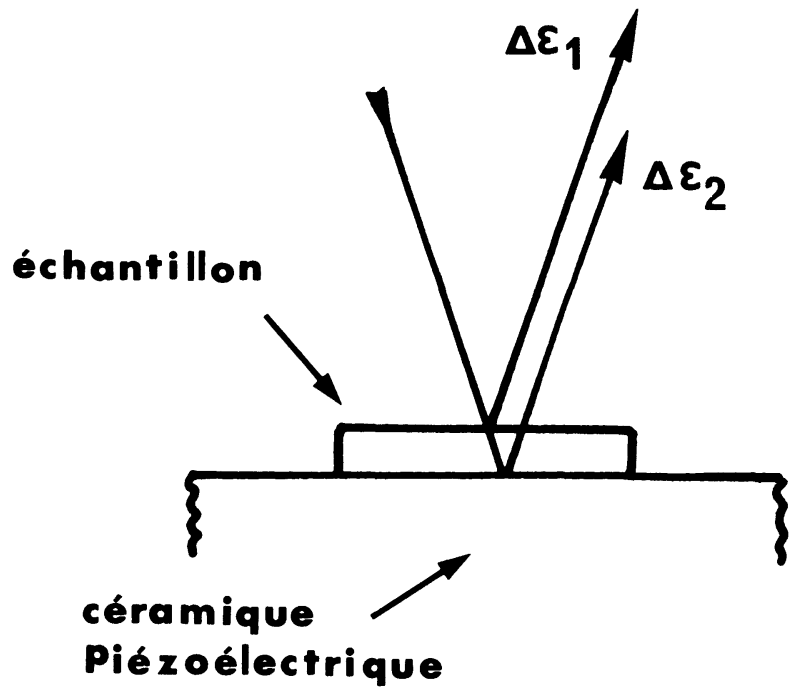

FIG. 1.

Le faisceau réfléchi sur la face arrière de l'échantillon traverse deux fois le matériau et contient une information proportionnelle aux propriétés d'absorption de ce matériau, c'est-à-dire à $\varepsilon_{2}$. Dans la mesure où le gap indirect est inférieur au gap direct, ce faisceau de rétrotransmission contient une information proportionnelle au coefficient de piézotransmission au niveau du gap indirect :

$$
\left(\frac{\Delta R}{R}\right)_{\mathrm{ar}}=-\beta^{\prime} \Delta \varepsilon_{2} \simeq-\frac{\mathrm{d} \varepsilon_{2}}{\mathrm{~d} E} \Delta E_{\text {indirect }} .
$$

Soit en définitive :

$$
\frac{\Delta R}{R}=\alpha \Delta \varepsilon_{1}-\beta^{\prime} \Delta \varepsilon_{2} .
$$

Les allures et les phases respectives des signaux de piézoréflectance obtenus sur un échantillon mince à deux faces polies sont représentées sur la figure 2 . Il est

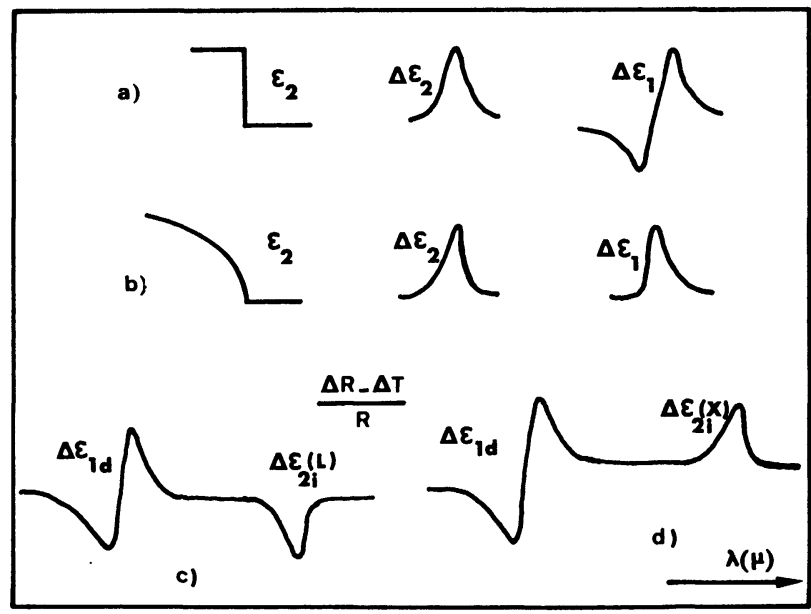

FIG. 2. - Illustration des allures des différentes structures : a) allure théorique du spectre de piézoréflectance au voisinage d'un gap direct excitonique. $b$ ) Allure théorique d'un spectre de piézo-transmission au voisinage du gap indirect excitonique. c) d) allures théoriques des spectres de piézo-réflexion-rétrotransmission dus à la succession d'un gap indirect et d'un gap direct. c) Gap indirect en $L, d$ ) gap indirect en $X$. 
ainsi évident que la position et l'identification du gap direct sont révélées par une structure présentant un passage par zéro, la position du gap indirect est révélée par une structure de pic et enfin que la nature du gap indirect est révélée par la phase relative de cette structure par rapport à la structure du gap direct.

A titre d'illustration, la figure 3 représente 3 types de spectres expérimentaux particulièrement caractéris-

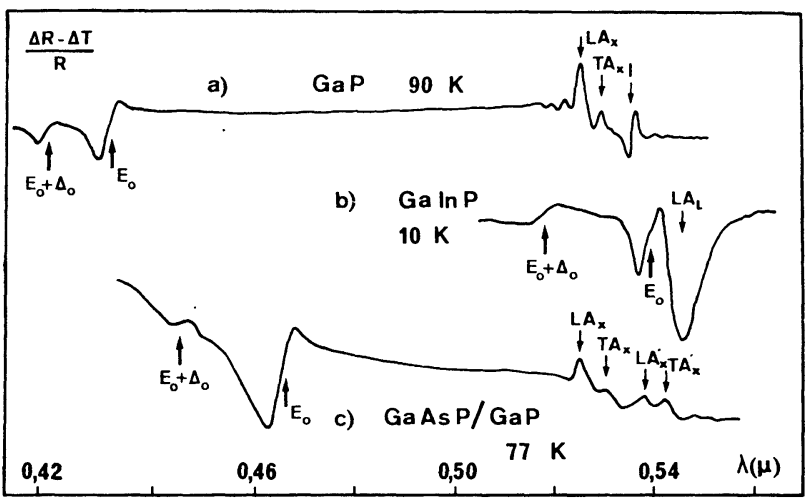

Fig. 3. - Spectres de piézo-réflexion-rétrotransmission de divers échantillons. a) $\mathrm{GaP}, \quad b) \mathrm{Ga}_{x} \mathrm{In}_{1-x} \mathrm{P} \quad(x=0,72)$, c) $\mathrm{GaAs}_{1-x} \mathrm{P}_{x}(x=0,90)$.

tiques. Le spectre $a$ ) représente le signal de piézoréflexion-rétrotransmission de GaP. Il est bien connu que le GaP est un semiconducteur indirect en $X$, nous observons un ensemble structures analogue à celui représenté sur la figure $2 d$, le gap direct et le gap indirect sont modulés en opposition de phase. La structure correspondant au gap direct $E_{0}$ est excitonique et a la forme représentée sur la figure $2 d$, les structures corres- pondant au gap indirect sont parfaitement marquées et tout à fait analogues aux structures que l'on observe sur un spectre de piézotransmission de $\mathrm{GaP}$, leur interprétation est donnée dans la référence [2].

Le spectre $b$ ) représente le signal observé sur un échantillon de $\mathrm{Ga}_{x} \operatorname{In}_{1-x} \mathrm{P} \quad(x=0,72)$. Une étude détaillée de cet alliage [4] nous a montré qu'il présentait un double croisement de bande et qu'en particulier pour $0,65<x<0,78$ il était indirect en $L$. L'ensemble des structures observées sur ce spectre est tout à fait analogue à celui de la figure $2 c$, les structures correspondant aux gaps direct $\left(E_{0}\right)$ et indirect $\left(\mathrm{LA}_{L}\right)$ sont modulées en phase.

Enfin le spectre $c$ ) représente le signal obtenu sur un échantillon de $\mathrm{GaAs}_{1-x} \mathrm{P}_{x}(x=0,90)$ épitaxié sur substrat de GaP. La couche épitaxiale est de l'ordre de $25 \mu \mathrm{m}$, le substrat a été aminci de manière à obtenir une structure finale constituée d'environ $25 \mu \mathrm{m}$ de GaAsP et $25 \mu \mathrm{m}$ de GaP. Le spectre de piézo-réflexion-rétrotransmission fait alors apparaître trois ensembles de structures, la structure correspondant au gap direct de l'alliage $\left(E_{0}\right)$, les structures caractéristiques du gap indirect de $\mathrm{GaP}\left(\mathrm{LA}_{x}\right.$ et $\left.\mathrm{TA}_{x}\right)$ et enfin les structures caractéristiques du gap indirect de l'alliage $\left(\mathrm{LA}_{x}^{\prime}\right.$ et $\left.\mathrm{TA}_{x}^{\prime}\right)$. Contrairement au précédent, cet alliage reste indirect en $X$ dans toute la gamme de concentration pour lequel il est indirect.

Les spectres de la figure 3 mettent clairement en évidence quelques exemples de résultats que l'on peut obtenir, notamment dans la caractérisation des alliages, par l'association des propriétés spécifiques de la piézomodulation et d'une configuration expérimentale originale.

\section{Bibliographie}

[1] Voir par exemple : Solid State Physics (Academic, New York, 1969), Vol. 11 Suppl. ; Semicond. and Semimetals 8, 9 (1972) ; Auvergne, D., CAMASSEL, J., Thèse d'Etat 1974.

[2] Auvergne, D., Merle, P., Mathieu, H., Phys. Rev. 12 (1975) 1371.

[3] Mathieu, H., Auvergne, D., Merle, P. and Rustagi, K. C., Phys. Rev. B 12 (1975) 5846.

[4] Merle, P., Auvergne, D., Mathieu, H. and Chevallier, J., Phys. Rev. (à paraître).
[5] Montgomery, H., Proc. R. Soc. A 309 (1969) 521.

[6] Mathieu, H., Auvergne, D. and Merle, P., Phys. Stat. Sol. B 72 (1975) 609.

[7] Elliott, R. J., Phys. Rev. 108 (1957) 1374.

[8] Camphausen, D. L., Neville Connel, G. A. and Paul, W., Phys. Rev. Lett. 26 (1971) 184.

[9] Galissot, F. et Vergne, R., Publ. Scient. et Techn. du Minist. del'Air 412 (1965) 50. 